\title{
Rapidly exacerbating tuberculosis
}

\author{
Julia Röper-Kelmayr ${ }^{1}$, Klaus Weiglein ${ }^{2}$, Bernd Lamprecht ${ }^{2,3}$ and Franz A. Fellner ${ }^{1,4}$ \\ ${ }^{1}$ Central Radiology Institute, Kepler University Hospital, Medical Faculty of the Johannes Kepler University, Linz, Austria \\ ${ }^{2}$ Department of Pulmonology, Kepler University Hospital, Medical Faculty of the Johannes Kepler University, Linz, Austria \\ ${ }^{3}$ Paracelsus Medical University, Salzburg, Austria \\ ${ }^{4}$ Medical Faculty of the Friedrich-Alexander-University of Erlangen-Nürnberg, Germany
}

Even in developed countries tuberculosis still contributes to morbidity and mortality [1]. We report the case of a previously healthy man who experienced bronchogenic spread of tuberculosis. A plain radiogram of the chest was acquired in the course of a pre-employment examination. This revealed multiple patchy infiltrates in both lungs, especially in the superior lobes. At that time the patient was without clinical symptoms.
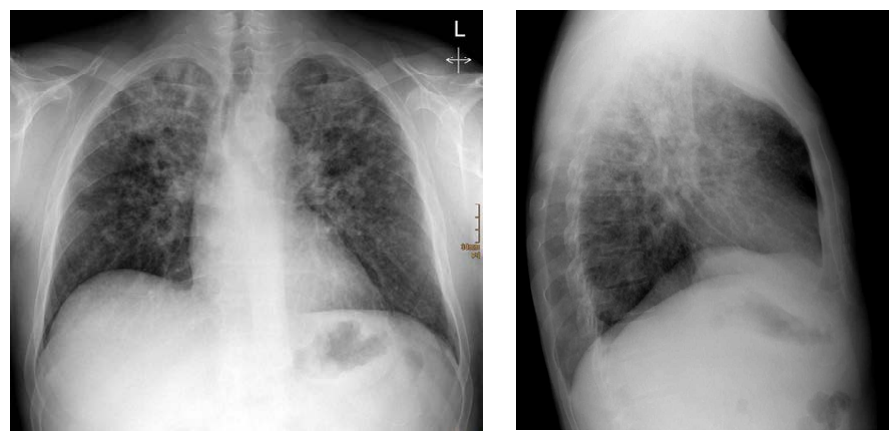

Figure 1. Plain radiogram of the chest shows multiple patchy infiltrations, especially in the superior lobes.

The patient was advised to consult a pulmonary specialist right away. Furthermore, a complementary CT examination of the chest was strongly recommended. However, the patient refused and left the hospital. 4 months later he came back with significant dyspnea, increasing malaise and was admitted to the department of pulmonology. Repeat X-ray as well as complementary computed tomography scans of
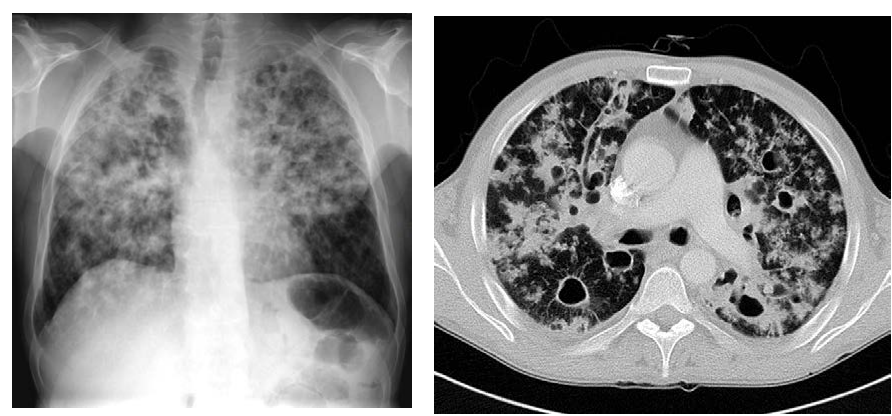

Figure 2. Follow-up chest radiogram and consecutive CT examination of the thorax reveal considerably increasing infiltrations, especially in the upper and middle fields. Moreover, CT shows multiple cavernizations. the chest demonstrated a considerable increase of the lung infiltrations in the upper and middle fields of both lungs.

Due to these findings, the suspected diagnosis was tuberculosis. This suspicion was finally confirmed by pulmonological examinations including tuberculin skin testing, interferon-gamma release assays and mycobacterial culture from bronchoalveolar lavage [2]. An adequate antituberculoustherapy using four drugs (isoniazid, rifampin, pyrazinamide, and ethambutol) was initiated [3,4]. A follow-up radiogram was acquired four weeks later that showed transition in a chronic destructive status with significant volume reduction and consecutive cranialization of the hili of the lungs.

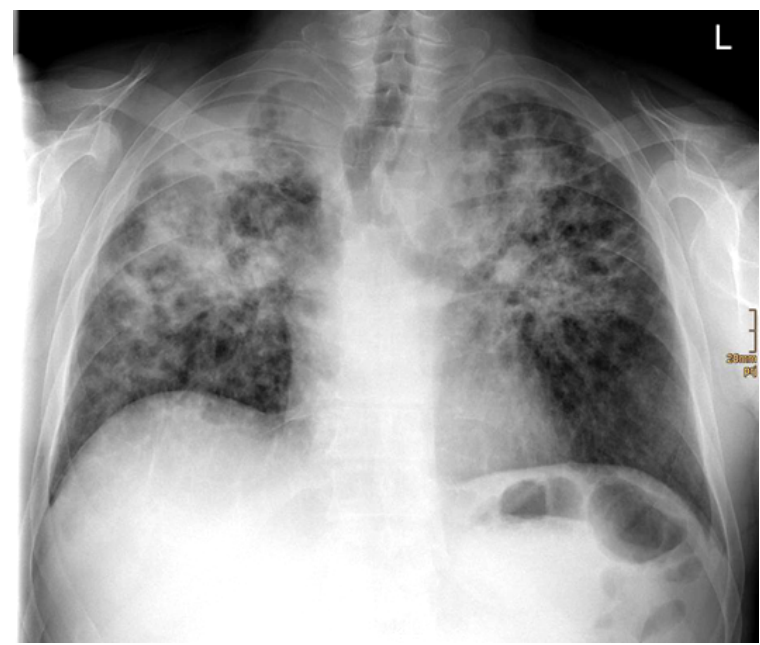

Figure 3. Follow-up chest radiography 4 weeks after initiation of therapy revealing a chronic destructive status with cranialization of the hili.

\section{References}

1. Geiss HK, Feldhues R, Niemann S, Nolte O, Rieker R (2005) Landouzy septicemia (sepsis tuberculosa acutissima) due to Mycobacterium microti in an immunocompetent man. Infection 33: 393-396.[Crossref]

2. Pai M, Nicol MP, Boehme CC (2016) Tuberculosis diagnostics: State of the art and future directions. Microbiol Spectr 4.[Crossref]

Correspondence to: Franz A. Fellner, Central Radiology Institute, Kepler University Hospital, Medical Faculty of the Johannes Kepler University, Linz, Austria, Medical Faculty of the Friedrich-Alexander-University of Erlangen Nürnberg, Germany, E-mail: franz.fellner@kepleruniklinikum.at

Received: March 03, 2017; Accepted: March 13, 2017; Published: March 16, 2017 
3. Nahid P, Dorman SE, Alipanah N, Barry PM, Brozek JL, et al. (2016) Official American thoracic society/centers for disease control and prevention/infectious diseases society of America clinical practice guidelines: Treatment of drug-susceptible tuberculosis.
Clin Infect Dis 63:e147-e195.[Crossref]

4. Dheda K, Barry CE 3rd, Maartens G (2016) Tuberculosis. Lancet387:1211-1226. [Crossref]

Copyright: (C2017 Röper-Kelmayr J. This is an open-access article distributed under the terms of the Creative Commons Attribution License, which permits unrestricted use, distribution, and reproduction in any medium, provided the original author and source are credited. 